\title{
ASSOCIAC̣ÕES ENTRE LARVAS DE COCHLIOMYIA MACELLARIA (FABRICIUS) E CHRYSOMYA ALBICEPS (WIEDEMANN) (DIPTERA, CALLIPHORIDAE) EM CONDIC̣ÕES EXPERIMENTAIS
}

\author{
Valéria M. Aguiar-Coelho ${ }^{1}$ \\ Margareth M.C. Queiroz ${ }^{3}$ \\ Eliane M.V. Milward-de-Azevedo ${ }^{2,3}$
}

\begin{abstract}
ASSOCIATIONS BETWEEN COCHIIOMYIA MACELlaRIA (FABRICIUS) AND Chrysomya albiceps (WIEDemann) Larvae (CALliphoridae, Diptera), IN EXPERIMENTAL CONDITIONS. This paper has the purpose of studying the interspecif relationships of larvae of Cochliomyia macellaria (Fabricius) and Chrysomya albiceps (Wiedemann) in laboratory. The experiments were conducted on climatized chamber set at $30^{\circ} \mathrm{C}, 60+10 \%$ UR and $14 \mathrm{~h}$ photophase. The relation of 1 larva/g diet was established. The association influence of post-embrionary development duration of both species. The association with Chrysomya albiceps influenced the weight of mature larvae of Cochliomyiia macellaria, reducing it significantly. Such weight decrease, along with the predation of larvae of $C$. macellaria larvae, yielded a decrease in survival in all of the stages.

KEY WORDS. Blowfly, interspecific relationship, larval development, predation
\end{abstract}

O estabelecimento de espécies de Chrysomya (Robineau-Desvoidy) no Novo Mundo têm afetado muscóides da fauna nativa provocando o seu deslocamento ou extinção em determinado nicho. HANSKI (1976) sugeriu que Lucilia caesar (Linnaeus) foi extinta das Ilhas Canárias devido à competição com Chrysomya albiceps (Wiedemann). BAUnGaRTNER \& GREENBERG (1984) sugeriram que $C$. albiceps e C. putoria (Wiedemann), espécies de relevante importância sócioeconômica (FurlanetTo et al. 1984; GREENBERG 1988; LAWSON \& GeMMEL 1990), estão envolvidas na supressão de Cochliomyia macellaria (Fabricius), no Peru. No Brasil, Ferreira (1983) e Mendes \& Linhares (1993) assinalaram que C. macellaria provavelmente foi deslocada de Goiânia e Campinas após a introdução de Chrysomya putoria. No Rio de Janeiro, D'ALMEIDA \& LOPES (1983) constataram o deslocamento de Cochliomyia macellaria de uma área urbana para a área rural. Segundo BAUnGARTNER \& GREENBERG (1984), a competição entre moscas varejeiras é provavelmente mais intensa durante o estágio larval. Visando subsidiar a compreensão destas relações, objetivou-se estudar as associações entre

1) Departamento de Patologia e Imunologia, Universidade de Nova Iguaçu. 26260-0)(00 Nova Iguaçu, Rio de Janeiro, Brasil.

2) Departamento de Parasitologia Animal, Universidade Federal Rural do Rio de Janeiro. Seropédica 23853-970 Itaguaí, Rio de Janeiro, Brasil.

3) Bolsista do CNPq.

Revta bras. Zool. 12 (4): $983-990,1995$ 
as fases imaturas de C. macellaria e Chrysomya albiceps, sob condições de laboratório, comparando-se os resultados obtidos em culturas mistas e puras.

\section{MATERIAL E MÉTODOS}

O estabelecimento, a manutenção e os procedimentos relativos à criação de Cochliomyia macellaria e Chrysomya albiceps seguiram a metodologia descrita por Aguiar-Coelho \& Milward-De-Azevedo (no prelo), Cunha-E-Silva \& Milward-DE-AzEvedo (1994) e Queiroz \& Milward-DE-AZEvedo (1991). Durante a etapa experimental, utilizou-se espécimens da quarta e terceira geração, respectivamente. Inoculou-se 1 larva/g de dieta à base de carne equina em decomposição. A carne equina, previamente fresca e descongelada, foi mantida durante 11 dias em refrigerador regulado a $12^{\circ} \mathrm{C}$ de temperatura.

Monitorou-se, paralelamente, o desenvolvimento dos insetos em culturas mistas e em culturas puras. Cada tratamento constou de quatro repetições. Para verificar-se o efeito da associação, transferiu-se 25 neolarvas de cada espécie, num total de 50 neolarvas, para um frasco $(4,0 \mathrm{~cm}$ de diâmetro por $5,5 \mathrm{~cm}$ de altura), contendo $50 \mathrm{~g}$ de dieta. No tratamento que caracterizou-se pela ausência da associação inter-específica, foram agrupadas 50 neolarvas de cada espécie em frascos contendo $50 \mathrm{~g}$ de dieta. Estes frascos foram introduzidos em recipientes plásticos maiores $(5,0 \mathrm{~cm}$ de diâmetro por $6,0 \mathrm{~cm}$ de altura), contendo vermiculite e tampados com tecido de náilon. Após o abandono da dieta, as larvas foram individualizadas, pesadas e transferidas para tubos de ensaio $(2,0 \mathrm{~cm}$ de diâmetro por $12,0 \mathrm{~cm}$ de altura) contendo vermiculite e tampados com algodão hidrófugo.

$\mathrm{O}$ experimento foi conduzido em câmara climatizada regulada a $30^{\circ} \mathrm{C}$ de temperatura, $60 \pm 10 \%$ de umidade relativa e 14 horas de fotofase. As observações foram diárias.

Os resultados experimentais foram submetidos à prova não paramétrica de Kruskal-Walles (ZAR 1984). Foram verificados os efeitos de combinação e de espécie. Os valores $\mathrm{F}$ calculados foram considerados significativos quando $\mathrm{p}<0,05$. Quando $0,05<\mathrm{p}, 10$ foi referida tendência à significância. Os contrastes entre postos médios foram estimados através do cálculo da dms ao nível de $5 \%$.

\section{RESULTADOS E DISCUSSÃO}

A duração das diferentes fases de desenvolvimento pós-embrionário de Cochliomyia macellaria e Chrysomya albiceps, em cultura isolada e associada, está registrada na tabela I. Ao monitorar-se o efeito de espécie, verificou-se que, em cultura pura, o desenvolvimento pós-embrionário de $C$. albiceps foi significativamente mais reduzido que o de Cochliomyia macellaria, corroborando com os resultados evidenciados por CUNHA-E-SILVA \& MILWARD-DE-AZEVEDO (1994) e QUEIROZ \& MilWARD-DE-AZEVEDO (1991), que trabalharam em condições experimentais semelhantes.

Cochliomyia macellaria respondeu, significativamente, à associação com Chrysomya albiceps, reduzindo o tempo médio dispendido nas diferentes etapas 
Tabela I. Duração do desenvolvimento pós-embrionário de Cochliomyia macellaria e Chrysomya albiceps isoladas e em associação, criadas em dieta à base de carne eqüina em decomposição, sob condições controladas $\left(30^{\circ} \mathrm{C}\right.$, U.R. $60 \pm 10 \%, 14$ h de fotofase).

\begin{tabular}{|c|c|c|c|c|}
\hline \multirow{3}{*}{ Espécies } & \multicolumn{4}{|c|}{ Duraçāo (dias) } \\
\hline & Neolarvas ao abandono & Estágio larval & Estágio pupal & Neolarvas a adultos \\
\hline & $x+5 x$ & $x+S \bar{x}$ & $x+5 x$ & $x+5 x$ \\
\hline \multicolumn{5}{|l|}{ ISOLADA } \\
\hline C. macellaria & $4,38+0,24 \mathrm{Aa}$ & $5,38+0,24 \mathrm{Aa}$ & $4,20 \pm 0,10 \mathrm{Aa}$ & $9,55+0,29 \mathrm{Aa}$ \\
\hline C. albiceps & $4,02 \pm 0,02 \mathrm{Ab}$ & $5.02+0.02 \mathrm{Ab}$ & $3,93 \pm 0,06 \mathrm{Ab}$ & $8.95 \pm 0.06 \mathrm{Ab}$ \\
\hline \multicolumn{5}{|l|}{ ASSOCIADA } \\
\hline C. macellaria & $3,60 \pm 0,28$ ba & $4,75+0,29 \mathrm{Ba}$ & $3,37+0,38 \mathrm{Ba}$ & $8,04 \pm 0,03 \mathrm{Ba}$ \\
\hline C. albiceps & $3,83+0,25 \mathrm{Aa}$ & $4,83+0,25 \mathrm{Aa}$ & $3,98 \pm 0,01 \mathrm{Ab}$ & $8,71+0,35 \mathrm{Ab}$ \\
\hline
\end{tabular}

1) As interações foram analisadas através do teste $F$, ao nível de $5 \%$ de probabilidade.

2) Médias seguidas pelas mesmas letras (efeito de combinação: letra maiúscula; efeito de espécie: letra minúscula), não diferem entre si pelo método das diferenças mínimas significativas (dms).

Tabela II. Peso das larvas que abandonaram a dieta e taxas de sobrevivência de adultos de Cochliomyia macellaria e Chrysomya albiceps isoladas e em associação, criadas em dieta à base de carne eqüina em decomposição, sob condições controladas $\left(30^{\circ} \mathrm{C}\right.$, U.R. $60 \pm 10 \%, 14 \mathrm{~h}$ de fotofase).

\begin{tabular}{|c|c|c|c|c|}
\hline \multirow{2}{*}{ Espécies } & \multirow{2}{*}{$\frac{\text { Peso de larvas (mg) }}{\bar{x}+5 \bar{x}}$} & \multicolumn{3}{|c|}{ Sobrevivência $(\%)$} \\
\hline & & Larval & Pupal & Neolarvas a adultos \\
\hline \multicolumn{5}{|l|}{ ISOLADA } \\
\hline C. macel/aria & $67,43+0,69 \mathrm{Aa}$ & $97,50 \mathrm{Aa}$ & $84,13 \mathrm{Aa}$ & $82,00 \mathrm{Aa}$ \\
\hline C. albiceps & $79,49+4,87 \mathrm{Ab}$ & $95,00 \mathrm{Aa}$ & $91,06 \mathrm{Ab}$ & $86,00 \mathrm{Ab}$ \\
\hline \multicolumn{5}{|l|}{ ASSOCIADA } \\
\hline C. macellaria & $47,92 \pm 2,09 \mathrm{Ba}$ & $21,33 \mathrm{Ba}$ & $80,55 \mathrm{Aa}$ & $16,00 \mathrm{Ba}$ \\
\hline C. albiceps & $69,79 \pm 6,64 \mathrm{Bb}$ & $91,00 \mathrm{Ab}$ & $89,30 \mathrm{Ab}$ & $82,67 \mathrm{Ab}$ \\
\hline
\end{tabular}

1) As interações foram analisadas através do teste $F$, ao nivel de $5 \%$ de probabilidade.

2) Médias seguidas pelas mesmas letras (efeito de combinação: letra maiúscula; efeito de espécie: letra minúscula), não diferem entre si pelo método das diferenças mínimas significativas (dms).

de seu desenvolvimento. Por outro lado, Chrysomya albiceps não alterou significativamente a sua velocidade de desenvolvimento ao associar-se com a espécie autóctone. O início do abandono das larvas da dieta, nas duas populações amostradas, foi mais precoce em cultura mista (Fig. 1). Esta resposta foi, provavelmente, induzida, entre outros fatores, pelas modificações físicas e nutricionais provocadas no meio de criação (HANSKI 1987a,b; PUTMAN 1983; DENNO \& COTHRAN 1975) e pode ter contribuído para a redução significativa do peso médio de larvas logo após o seu abandono da dieta (Tab. II). Esta redução foi de cerca de 20 e $10 \mathrm{mg}$ para Cochliomyia macellaria e Chrysomya albiceps, respectivamente. A modificação da microbiota inferida por quaisquer das duas espécies envolvidas na associação pode ser responsabilizada por estas modi- 


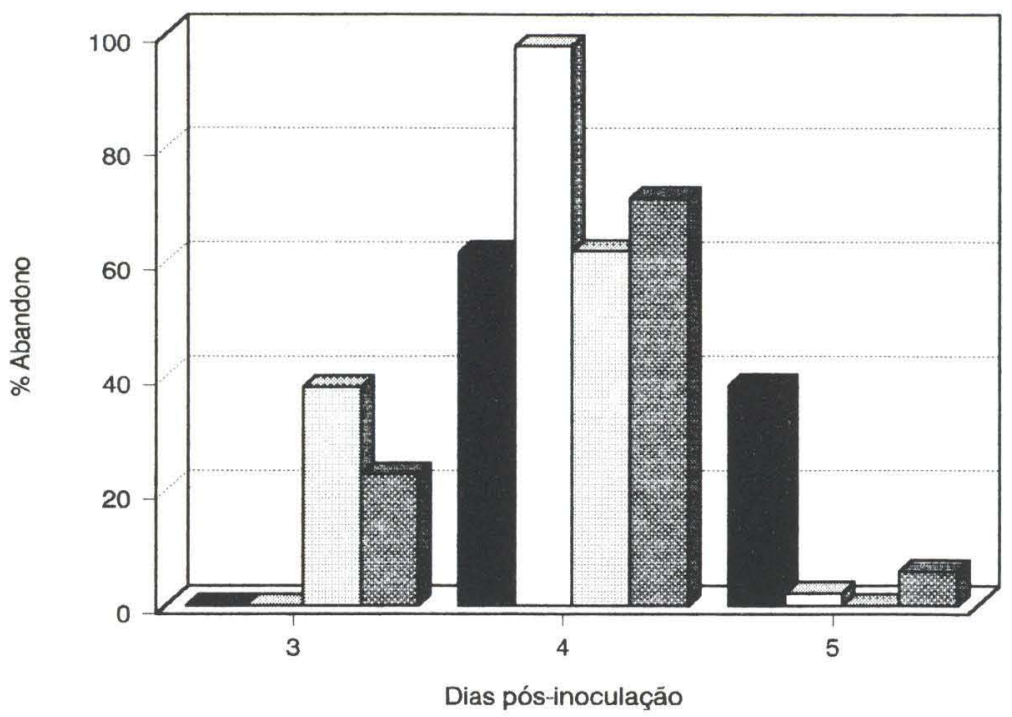

Co. macellaria (I) $\square$ Ch. albiceps (I) $\square$ Co. macellaria (A) $\$$ Ch. albiceps (A)

Fig. 1. Ritmo de abandono de larvas de Cochliomyia macellaria e Chrysomya albiceps de uma dieta à base de carne eqüina, em culturas isoladas (I) e associadas (A), sob condições controladas $\left(30^{\circ} \mathrm{C}\right.$, U.R. $60 \pm 10 \%, 14 \mathrm{~h}$ de fotofase $)$.

ficações. Os insetos detritívoros, e entre eles, os sapronecrófagos, são significativamente influenciados pelos microorganismos associados à dieta (PUTMAN 1983; SLANSKY \& SCRIBER 1985; HANSKY 1987a). A possível liberação de alomônios por Chrysomya albiceps, motivando a "fuga" de larvas de outras espécies, é uma hipótese que também deve ser considerada e testada. CUNHA-E-SILVA, MiLwARDDE-AZEVEDO, FREITAS e QUEIROZ (observações não publicadas) após conduzirem, com sucesso, culturas puras de Cochliomyia macellaria por oito gerações sucessivas, verificaram que, durante a nona geração, as larvas desta espécie reagiram atípica e desordenadamente, quando introduziu-se, na mesma câmara de criação, recipientes contendo espécimens imaturos de Chrysomya albiceps. É interessante observar que a introdução de larvas de $C$. megacephala nesta câmara, anteriormente, não provocara reação similar. Embora a problemática relacionada à concentração excessiva de amônia e de outros gazes eliminados durante o processo de putrefação da carne, em ambientes confinados, não deva ser desprezada, especulou-se sobre a possível liberação de alomônios pela espécie invasora.

A taxa de sobrevivência de larvas de Cochliomyia macellaria, em cultura mista, foi de apenas 21,3\%. Em cultura pura, registrou-se a pupariação de 97,5\% dos espécimens. CUnHA-E-Silva \& MiLward-DE-AZEVEDo (1994) obtiveram cerca de $75 \%$ de pupas formadas à partir de larvas também criadas em culturas puras e que apresentavam o peso final entre 47,0 e 56,0 mg. Foi evidenciada a predação de larvas de Cochliomyia macellaria por larvas de Chrysomya albiceps 
fora da dieta. Esta foi quantificada pelos resíduos de larvas, caracterizados por cutículas ressecadas, num percentual que variou de 33,3 a $100 \%$, entre as diferentes repetições. Ao associarem larvas de Cochliomyia macellaria e Chrysomya rufifacies, WELLS \& GREENBERG (1992a,b) verificaram, como no presente trabalho, que o terceiro ínstar de Cochliomyia macellaria foi o mais atingido pela predação. A redução das populações de moscas varejeiras, no campo, determinada pela atividade de predadores e parasitóides, é mais evidente durante a emigração das larvas maduras da carcaça, e no estágio de pupa (FULLER 1934; Khole 1978; Levot et al. 1979; Blackith \& Blackith 1990). Carcaças de coelhos expostas ao meio ambiente induziram, significativamente, uma maior colonização por Cochliomyia macellaria na ausência de Chrysomya rufifacies sugerindo a atividade predatória facultativa por esta espécie (WELLS \& GREENBERG 1992c). MARCHENKO (1985) descreveu detalhadamente o comportamento canibalista e predatório de $C$. albiceps e destacou que, na ausência de carne, esta espécie pode completar o seu desenvolvimento alimentando-se de larvas de outros dípteros. Por outro lado, o efeito provocado pelo procedimento metodológico, adotado no presente bioensaio, deve ser considerado. O confinamento dos espécimens nos pequenos recipientes contendo vermiculite, impediu a expressão comportamental espontânea das larvas maduras de Cochliomyia macellaria. Estas larvas, na natureza, podem afastar-se consideravelmente da fonte de alimento, enterrando-se no solo logo a seguir (GREENBERG 1990). Este mecanismo inclui uma estratégia de proteção contra predadores e/ou parasitóides.

$\mathrm{O}$ aumento da atividade motora de Chrysomya albiceps devido à sua característica predatória, por um lado, e, por outro, à reação de escape de Cochliomyia macellaria, provavelmente comprometeram o tempo de consumo e utilização dos nutrientes ingeridos por estas espécies. Essas atividades podem, assim, também explicar a redução do peso de larvas maduras dos espécimens comprometidos com a associação. O custo metabólico determinado pela movimentação das larvas, na dieta, e provocada pela ação de captura e fuga, inerente ao processo predatório, foi destacado por SLANSKY \& SCRIBER (1985).

Os adultos provenientes das duas populações, e oriundos de larvas criadas em cultura mista, iniciaram o processo de emergência mais precocemente (Fig.2).

As taxas de sobrevivência de pupas das duas espécies não foi influenciada pela associação. Apenas $16 \%$ das larvas de Cochliomyia macellaria inoculadas em cultura mista originaram adultos. As taxas de sobrevivência das diferentes fases de desenvolvimento de Chrysomya albiceps não apresentaram diferenças significativas entre os tratamentos (Tab. II).

Este experimento, portanto, corrobora com a hipótese apresentada por FERREIRA (1983) e reiterada por MENDES \& LinHARES (1993): C. albiceps tem participado ativamente do deslocamento da espécie autóctone, Cochliomyia macellaria, em diferentes localidades da América do Sul. Por outro lado, o presente ensaio demonstrou que, sob as condições previstas, a associação entre as duas espécies monitoradas foi deletéria também para Chrysomya albiceps, ao produzir a redução significativa do peso corporal dos espécimens, interferindo, assim, diretamente sobre o potencial biótico dos adultos. 


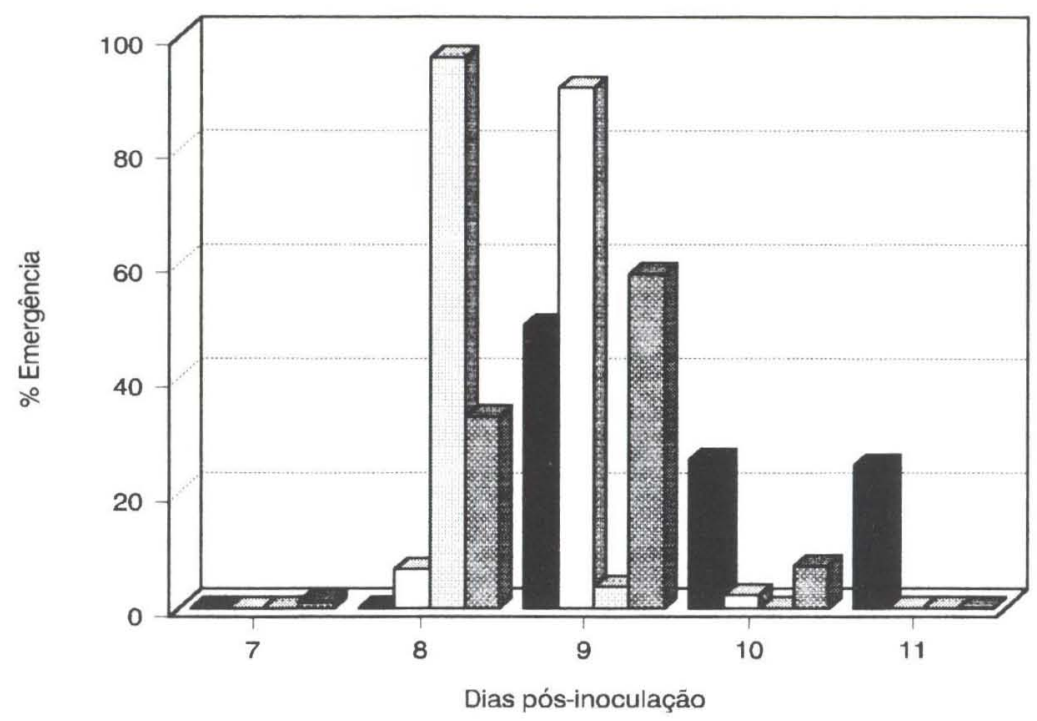

Co. macellaria (I) $\square$ Ch. albiceps (I) $\square$ Co. macellaria (A) $\$$ Ch. albiceps (A)

Fig. 2. Ritmo de emergência dos adultos de Cochliomyia macellaria e Chrysomya albiceps oriundos de larvas criadas em dieta à base de carne eqüina, em culturas isoladas (I) e associadas (A), sob condições controladas $\left(30^{\circ} \mathrm{C}\right.$, U.R. $60 \pm 10 \%, 14 \mathrm{~h}$ de fotofase).

AGRADECIMENTOS. Ao Prof. Paulo Roberto Curi, da Universidade Estadual Paulista, Botucatu (São Paulo), pela execução da análise estatística. A Coordenadoria de Aperfeiçoamento de Pessoal de Nível Superior (CAPES) e à FINEP/PCTPA/CNPq pelo suporte financeiro à primeira autora e ao trabalho.

\section{REFERÊNCIAS BIBLIOGRÁFICAS}

Aguiar-Coelho, V.M. \& E.M.V. Milward-DE-AZEvedo. (no prelo). Relações intra-específicas de Cochliomyia macellaria (Fabricius), Chrysomya albiceps (Wiedemann) e Chrysomya megacephala (Fabricius) (Diptera: Calliphoridae), em condições experimentais. Revta bras. Ent.

Baumgartner, D.L. \& B. Greenberg. 1984. The genus Chrysomya (Diptera: Calliphoridae) in the New World. Jour. Med. Entomol. 21: 105-113.

BLACKITH, R.E. \& R.M. BLACKITH. 1990. Insect infestations on small corpses. Jour. Nat. Hist. 24: 699-709.

Cunha-E-Silva, S.L. \& E.M.V. Milward-DE-AzEvedo. 1994. Estudo comparado do desenvolvimento pós-embrionário de Cochliomyia macellaria (Fabricius) (Diptera, Calliphoridae) em duas dietas à base de carne, em laboratório. Revta bras. Zool. 11: 659-688.

D’Almeida, J.M. \& H.S. DE LOPES. 1983. Sinantropia de Dípteros Caliptratos (Calliphoridae) no Estado do Rio de Janeiro. Arq. Univ. Fed. Rur. Rio de 
Janeiro 6: 31-38.

DENNO, R.F. \& W.R. Cothran. 1975. Niche relationships of a guild of necrophagous flies. Ann. Ent. Soc. Amer. 68: 741-754.

FerreirA, M.J.M. 1983. Sinantropia de Calliphoridae (Diptera) em Goiânia, Goiás. Rev. Bras. Biol. 43: 199-210.

Fuller, M.E. 1934. The insects inhabitants of carrion: a study in animal ecology. Council Sci. Ind. Res. Australia Bull. 82: 1-62.

FurlanetTo, S.M.P.; M.L.C. CAmpos \& C.M. HARSI. 1984. Microorganismos enteropatogênicos em moscas africanas pertencentes ao gênero Chrysomya (Diptera, Calliphoridae) no Brasil. Revta Microbiol. 15: 170-174.

GreenberG, B. 1988. Chrysomya megacephala (F.) (Diptera: Calliphoridae) collected in North America and notes on Chrysomya species present in the New World. Jour. Med. Entomol. 25 (3): 199-200.

1990. Behavior of postfeeding larvae of some Calliphoridae and a Muscid (Diptera). Ann. Entomol. Soc. Am. 83: 1210-1214.

HANSKI, I. 1976. Breeding experiments with carrion files (Diptera) in natural conditions. Ann. Ent. Fenn. 42: 113-121.

1987a. Carrion fly community dynamics: patchiness, seasonality and coexistence. Ecol. Ent. 12: 257-266.

1987b. Nutritional ecology of dung and carrion feeding insects, p. 838-884. In: F. SlanSKy JR. \& J.G. RODRIGUEZ (Eds). Nutritional Ecology of Insects, mits, stidery and related Invertebrates. New York, J. Wiley \& Sons, $1016 \mathrm{p}$.

KHole, V. 1978. Studies on the population dynamics in larval blowflies (Calliphoridae: Diptera). Biovigyanam 4: 151-158.

LAwSON, J.R. \& M.A. GEMmELL. 1990. Transmission of taeniid tapeworm eggs via blowflies to intermediate hosts. Parasitology 100: 143-146.

LEVOT, G.W.; K.R. BRown \& E. ShIPP. 1979. Larval growth of some calliphorid and sarcophagid Diptera. Bull. Entomol. Res. 69: 469-475.

MARChENKO, M.I. 1985. Development of Chrysomya albiceps Wd. (Diptera, Calliphoridae). Entomol. Obozr. 64: 79-84.

Mendes, J. \& A.X. Linhares. 1993. Atratividade por iscas e estágios de desenvolvimento ovariano em várias espécies sinantrópicas de Calliphoridae (Diptera). Revta bras. Ent. 37: 157-166.

PUTMAN, R.J. 1983. Carrion and dung: the decomposition of animals wastes. Studies in Biology no. 156. Edward Arnold. 60 p.

Queiroz, M.M.C. \& E.M.V. Milward-DE-AzEvedo. 1991. Técnicas de criação e alguns aspectos da biologia de Chrysomya albiceps (Wiedemann) (Diptera, Calliphoridae), em condições de laboratório. Revta bras. Zool. 8: 75-84.

SlANSKY JR., F. \& M. SCRIBER. 1985. Food consumption and utilization, p. 87-163. In: M.G.A. KERKUT \& L.I. GILBERT (Eds). Comprehensive Insect Physiology, Biochemistry and Pharmacology. Oxford, Pergamon Press, $162 \mathrm{p}$. 
WELLS, J.D. \& B. GREENBERG. 1992a. Laboratory interaction between introduced Chrysomya rufifacies and native Cochliomyia macellaria (Diptera: Calliphoridae). Environ. Entomol. 21: 640-645. . 1992b. Rates of predation by Chrysomya rufifacies (Macquart) on Cochliomyia macellaria (Fabr.) (Diptera: Calliphoridae) in the laboratory: effect of predator and prey development. Pan-Pac. Entomol. 68: 12-14.

1992c. Interaction between Chrysomya rufifacies and Cochiomyia macellaria (Diptera: Calliphoridae): the possible consequences of an invasion. Bull. Entomol. Res. 82: 133-137.

ZAR, J.H. 1984. Biostatistical Analizys. Englewood Cliffs, Prentice-Hall International Ed., $2^{\text {nd }}$ ed., 718 p.

Recebido em 24.VIII.1994; aceito em 19.IV.1996. 